Lecture Notes in Computational Vision and Biomechanics 34

João Manuel R. S. Tavares

Renato Manuel Natal Jorge Editors
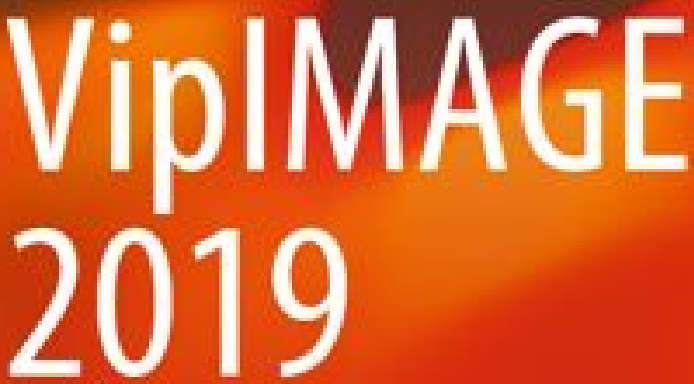

Proceedings of the VII ECCOMAS

Thematic Conference on Computational Vision and Medical Image Processing, October 16-18, 2019, Porto, Portugal

Q Springer 


\title{
Manufacturing process of a brain aneurysm bio model in PDMS using rapid prototyping
}

\author{
Andrews Souza ${ }^{1}$, João Ribeiro ${ }^{2}$, Rui Lima ${ }^{3}$ \\ 1 Polytechnic Institute of Bragança, Portugal, email: andrews.va.souza@alunos.ipb.pt \\ 2 Polytechnic Institute of Bragança, CIMO, Portugal, email: jribeiro@ipb.pt \\ 3 MEtRiCS, Mechanical Eng. Dep., University of Minho, Portugal. CEFT, Faculdade de \\ Engenharia da Universidade do Porto (FEUP), Portugal, email: rl@dem.uminho.pt
}

\section{Introduction}

Cerebral aneurysm or intracranial aneurysm is the abnormal dilatation of the blood vessel in a saccular form [1]. It may originate in congenital defects, weakening of the arterial wall with increasing age [2], atherosclerotic changes, trauma and infectious emboli. Other factors may be related to the onset of the aneurysm, such as hypertension, smoking, and excessive alcohol use. It is estimated that intracranial aneurysm occurs in $2.6 \%$ of the population and the cause of $85 \%$ of subarachnoid hemorrhage is the rupture of a saccular intracranial aneurysm [3].

In vivo experimental methods are an effective way to validate new practices and techniques for diseases such as the aneurysm, but in addition to ethical issues, these kinds of experiments are expensive and have low reproductively [4]. Thus, in order to better understand the pathophysiological and geometric aspects of an aneurysm, it is important to manufacture in vitro models to improve existing endovascular treatments and to develop and validate theoretical and computational models [5-6].

Recently Pinho et al developed a model with geometry and dimensions based on clinical data of a common saccular intracranial aneurism. The model was fabricated with the help of a 3D printer and produced with the polydimethylsiloxane (PDMS) which is a biocompatible material [7]. The PDMS has an elastic behavior similar to arteries [8] and with numerous applications in biomedical [9-12]. The model was subsequently subjected to different experimental tests and numerical simulations [2, 13].

In order to improve the existing model, in this work we propose a novel process of manufacturing a cerebral aneurysm biomodel obtained through an angiography. The image was processed by means of the Scanlp software and the mould was obtained by a three-dimensional printing process (TDP) combined with a PDMS gravity casting process. The biomodel fabricated in this work allows the performance of different hemodynamic studies, test different aneurysm repair technique and validate numerical approaches. 


\section{Manufacturing process}

The first process used to obtain the biomodel of the cerebral aneurysm was the angiography of the anatomical zone where the aneurysm is located. For the image treatment, it was performed the segmentation of the image with the objective to isolate the internal carotid artery from the others structures presented in the imaging examination.

The software used was Scanlp, which read and import the angiography images, translating to DICOM (Digital Imaging and Communications in Medicine) format, allowing the visualization of the $3 \mathrm{D}$ image. In order to isolate the structure of interest, we used the technique of binarization (Thresholding) and the thickness of the layer (Thickness layer) was 0.0889 . Note that, it was the smallest available thickness and as a result it was obtained the best possible precision. In Fig. 1 we can visualize the image after this treatment step.

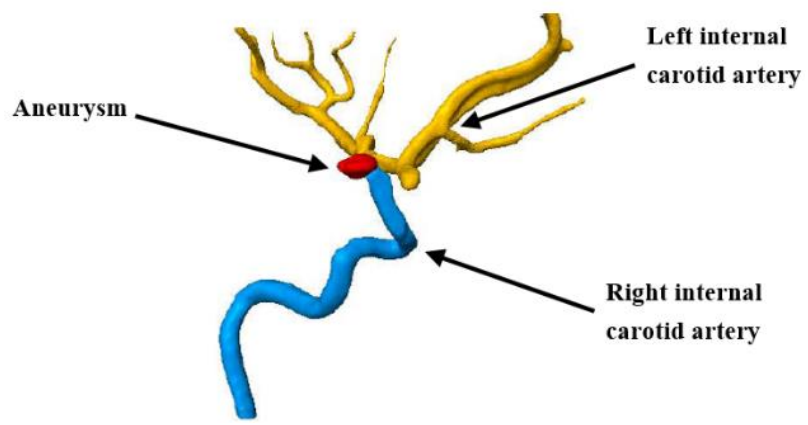

Fig. 1 Mask image processing step.

Finally, a mask was applied to the aneurysm and its arteries. After this procedure the images were converted to stl format. The 3D model has been converted to sli. (slice) to be laminated into thin slices parallel to each other and perpendicular to the $\mathrm{Z}$ axis. The file was generated and sent to the three-dimensional printer. 

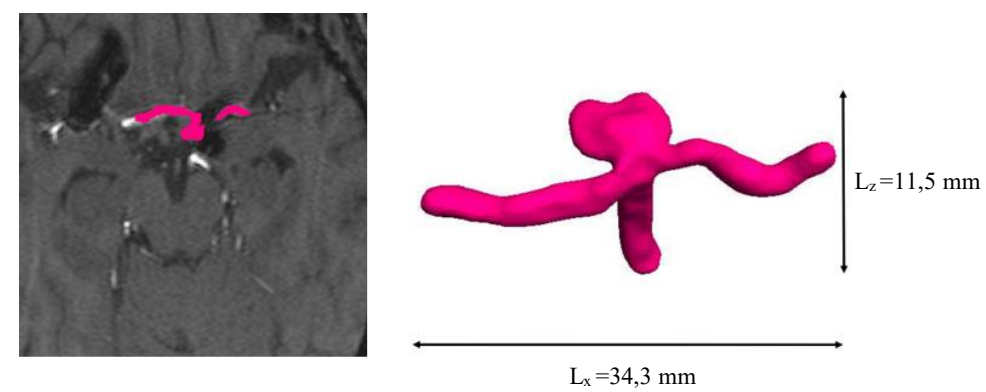

Fig. 2 Final model of the aneurysm

\section{Rapid prototyping}

Rapid Prototyping (RP) is a technology that builds physical structures layer by layer from a virtual model, these structures can be made of various materials and for a range of applications. This technology was initially developed for the manufacturing industry, designing components for automobiles, aircraft and computers. Rapid prototyping was first used in the field of medicine in 1990 when a model of cranial bone anatomy was produced demonstrating complete internal details with computed tomography image data [14]. At the present moment, industrial and academic interest in three-dimensional (3D) prints has grown and 3Dprinted products have been gaining space in pre-surgical planning and operative use [15].

In this work, this technology was used to generate the physical model of the aneurysm. For this, we used the 3D ProJet 1200 printer, this printer prints in wax, and it is possible to achieve a good finish.

\section{In vitro PDMS bio model}

With the finished wax physical model, we prepared 10:1 PDMS, i.e., 10 grams of the base polymer correspond to 1 gram of the curing agent. After, performing the mixture, it was used a vacuum pump for remove the bubbles and subsequently poured into the mold by means of a gravity casting process. The curing process was performed at room temperature for about 24 hours. At the end, the wax was carefully removed from the mold. 


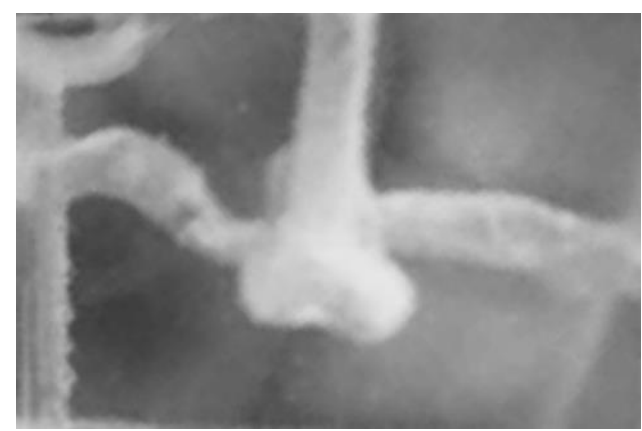

Fig. 3 Biomodel in PDMS with internal form of a cerebral aneurism

\section{Conclusions}

One of the major difficulties affecting the long-term success of aneurysm repair is the migration of the graft (stent), which may generate internal leakage and even rupture. To avoid this phenomenon and other problems related to the aneurysm treatment, there is the need to perform different kinds of tests, in order to improve techniques and materials, which is very difficult to be carried out in vivo due to the difficulty related to the organs authorization, high costs and reproducibility. The low cost of the in vitro model manufacturing process proposed in this study aims to help and simplify the test of aneurysms. This process is a simple method able to replicate different configurations of aneurysms, which can be used not only for medical and preoperative diagnosis but also to perform in vitro flow experiments to validate and improve existent numerical models.

\section{Acknowledgments}

This work was partially supported by Fundação para a Ciência e a Tecnologia (FCT) under the strategic grants UID/EMS/04077/2019 and UID/EMS/00532/2019. The authors are also grateful for the funding of FCT through the projects POCI-01-0145-FEDER-016861, NORTE-01-0145-FEDER029394, NORTE-01-0145-FEDER-030171, funded by COMPETE2020, NORTE2020, PORTUGAL2020, and FEDER.

\section{References}

[1] Rodriguez-Régent C (2014) Non-invasive diagnosis of intracranial aneurysms. Diagn. Interv. Imaging 95: 1163-1174 
[2] Cardoso C, Fernandes CS, Lima R, Ribeiro J (2018) Biomechanical analysis of PDMS channels using different hyperelastic numerical constitutive models. Mech. Res. Commun 90: 26-33

[3] Sathyan S (2014) Association of Versican (VCAN) gene polymorphisms rs251124 and rs2287926 (G428D), with intracranial aneurysm. Meta Gene 2: 651-660

[4] Bosman WMPF (2010) Aortic customize: An in vivo feasibility study of a percutaneous technique for the repair of aortic aneurysms using injectable elastomer. Eur. J. Vasc. Endovasc. Surg 40: 65-70

[5] Bosman WMPF(2010) The effect of injectable biocompatible elastomer (PDMS) on the strength of the proximal fixation of endovascular aneurysm repair grafts: An in vitro study. J. Vasc. Surg 52: 152-158

[6] Nam SW, Choi S, Cheong Y, Kim YH, Park HK (2015) Evaluation of aneurysm-associated wall shear stress related to morphological variations of circle of Willis using a microfluidic device. J. Biomech 48: 348-353

[7] Hassler C, Boretius T, Stieglitz T, (2011) Polymers for neural implants," J. Polym. Sci. Part B Polym. Phys 49: 18-33

[8] Lima R (2008) In vitro blood flow in a rectangular PDMS microchannel: Experimental observations using a confocal micro-PIV system," Biomed. Microdevices 10: 153-167

[9] Maitz MF (2015) Applications of synthetic polymers in clinical medicine. Biosurface and Biotribology 1: 161-176

[10] Unger MA, Chou HP, Thorsen T, Scherer A, Quake SR (2000) Monolithic microfabricated valves and pumps by multilayer soft lithography. Science 288: 113-6

[11] Bozukova D, Pagnoulle C, Jérôme R, Jérôme C (2010) Polymers in modern ophthalmic implants - Historical background and recent advances. Mater. Sci. Eng. R Reports 69: 63-83

[12] Wang W, Fang J (2007) Variable focusing microlens chip for potential sensing applications. IEEE Sens. J 7: 11-17

[13] Rodrigues RO, Pinho D, Bento D, Lima R, Ribeiro J (2016) Wall expansion assessment of an intracranial aneurysm model by a 3D Digital Image Correlation System. Measurement 88: 262 270

[14] Kim MS, Hansgen AR, Wink O, Quaife RA, Carroll JD (2008) Rapid prototyping: A new tool in understanding and treating structural heart disease. Circulation 117: 2388-2394

[15] Jakus AE, Rutz AL, Shah RN (2016) Advancing the field of 3D biomaterial printing. Biomed. Mater 11: 14102 